PROCEEDINGS OF THE

AMERICAN MATHEMATICAL SOCIETY

Volume 139, Number 10, October 2011, Pages 3497-3512

S 0002-9939(2011)10759-7

Article electronically published on February 9, 2011

\title{
EMBEDDING OF GLOBAL ATTRACTORS AND THEIR DYNAMICS
}

\author{
ELEONORA PINTO DE MOURA, JAMES C. ROBINSON, AND J. J. SÁNCHEZ-GABITES
}

(Communicated by Professor Yingfei Yi)

\begin{abstract}
Suppose that $\mathcal{A}$ is the global attractor associated with a dissipative dynamical system on a Hilbert space $H$.

If the set $\mathcal{A}-\mathcal{A}$ has finite Assouad dimension $d$, then for any $m>d$ there are linear homeomorphisms $L: \mathcal{A} \rightarrow \mathbb{R}^{m+1}$ such that $L \mathcal{A}$ is a cellular subset of $\mathbb{R}^{m+1}$ and $L^{-1}$ is log-Lipschitz (i.e. Lipschitz to within logarithmic corrections). We give a relatively simple proof that a compact subset $X$ of $\mathbb{R}^{k}$ is the global attractor of some smooth ordinary differential equation on $\mathbb{R}^{k}$ if and only if it is cellular, and hence we obtain a dynamical system on $\mathbb{R}^{k}$ for which $L \mathcal{A}$ is the global attractor. However, $L \mathcal{A}$ consists entirely of stationary points.

In order for the dynamics on $L \mathcal{A}$ to reproduce those on $L \mathcal{A}$ we need to make an additional assumption, namely that the dynamics restricted to $\mathcal{A}$ are generated by a log-Lipschitz continuous vector field (this appears overly restrictive when $H$ is infinite-dimensional, but is clearly satisfied when the initial dynamical system is generated by a Lipschitz ordinary differential equation on $\mathbb{R}^{N}$ ). Given this we can construct an ordinary differential equation in some $\mathbb{R}^{k}$ (where $k$ is determined by $d$ and $\alpha$ ) that has unique solutions and reproduces the dynamics on $\mathcal{A}$. Moreover, the dynamical system generated by this new ordinary differential equation has a global attractor $\mathcal{X}$ arbitrarily close to $L \mathcal{A}$.
\end{abstract}

\section{INTRODUCTION}

In this paper we discuss the problem of finding a finite-dimensional description of the asymptotic dynamics of a dissipative dynamical system on a real separable Hilbert space $H$ (with norm $\|\cdot\|$ ). The evolution of this dynamical system is described by a continuous semigroup $\{S(t)\}_{t \geq 0}$ of solution operators defined by

$$
S(t) u_{0}=u\left(t ; u_{0}\right), \text { for all } t \geq 0,
$$

where $u\left(t ; u_{0}\right)$ is the solution of the equation with initial condition $u_{0}$.

The long-term behaviour of the solutions of such a dynamical system is captured by its global attractor (Billotti and LaSalle, 1971).

Definition 1.1. Let $H$ be a Hilbert space, and let $S(t)$ be a continuous semigroup defined on $H$. A global attractor $\mathcal{A} \subset H$ is a compact invariant set, i.e. $S(t) \mathcal{A}=\mathcal{A}$

Received by the editors August 12, 2010 and, in revised form, August 23, 2010.

2010 Mathematics Subject Classification. Primary 37L30, 54H20, 57N60.

The first author is sponsored by CAPES and would like to thank CAPES for all their support during her Ph.D.

The second and third authors are supported by EPSRC Leadership Fellowship EP/G007470/1.

(C)2011 American Mathematical Society Reverts to public domain 28 years from publication 
for all $t \geq 0$, that attracts all bounded sets, i.e.

$$
\operatorname{dist}(S(t) B, \mathcal{A}) \longrightarrow 0 \quad \text { as } \quad t \longrightarrow \infty,
$$

for any bounded set $B \subset H$. If a global attractor $\mathcal{A}$ exists, then it is unique.

The distance in (1.1) is the Hausdorff semidistance between two non-empty subsets $X, Y \subset H, \operatorname{dist}(X, Y)=\sup _{x \in X} \inf _{y \in Y}\|x-y\|$.

This framework is particularly interesting for a class of dynamical systems generated by certain partial differential equations, for which the phase space $H$ is infinite-dimensional, but which have a global attractor that has finite box-counting dimension $\left(\operatorname{dim}_{\mathrm{B}}(\mathcal{A})<\infty\right)$. This is the case, for instance, for the KuramotoSivashinsky equation and the 2D Navier-Stokes equations (see Constantin and Foias (1988), Eden et al. (1994), Temam (1997), Robinson (2001) and Chepyzhov and Vishik (2002) for a more detailed study).

In this context it is natural to seek a finite-dimensional system of ordinary differential equations in some $\mathbb{R}^{m}$,

$$
\dot{x}=\mathcal{F}(x),
$$

whose asymptotic behaviour reproduces that of the original equation. Ideally,

(i) the attractor $\mathcal{A}$ would be embedded in $\mathbb{R}^{m}$ via some homeomorphism $L$ : $\mathcal{A} \longrightarrow L \mathcal{A} \subseteq \mathbb{R}^{m}$

(ii) the dynamics of (1.2) on $L \mathcal{A}$ would reproduce those of $S(\cdot)$ on $\mathcal{A}$, and

(iii) $L \mathcal{A}$ would be the global attractor for (1.2).

The existence of such a system of ordinary differential equations has only been proved for certain dissipative equations that possess an inertial manifold. Introduced by Foias et al. (1985), inertial manifolds are positively invariant finitedimensional Lipschitz manifolds that contain the global attractor and attract all trajectories at an exponential rate (see Constantin and Foias (1988), Constantin et al. (1989), Foias et al. (1988a), Foias et al. (1988b), Temam (1997), for more details). Foias et al. (1985) showed that if a certain spectral gap condition holds, then the system possesses an inertial manifold. Unfortunately this condition is very restrictive and there are many equations, such as the 2D Navier-Stokes equations, for which it is not satisfied. Thus it is desirable to adopt alternative approaches to the problem described above.

Following an approach pioneered by Eden et al. (1994), the main result of this paper is as follows. We discuss our assumptions after the statement of the theorem.

Theorem 1.2. Suppose that $S(\cdot)$ has a global attractor $\mathcal{A}$ such that

$$
d:=\operatorname{dim}_{\mathrm{A}}(\mathcal{A}-\mathcal{A})<\infty
$$

where $\operatorname{dim}_{\mathrm{A}}$ denotes the Assouad dimension. Assume that $\left.S(t)\right|_{\mathcal{A}}$ is generated by the differential equation

$$
\dot{u}=\mathcal{G}(u), \quad u \in \mathcal{A},
$$

where $\mathcal{G}$ is log-Lipschitz with exponent $\alpha<1 / 2$,

$$
\|\mathcal{G}(u)-\mathcal{G}(v)\| \leq C_{\mathcal{G}}\|u-v\|\left(\log \frac{R}{\|u-v\|}\right)^{\alpha} \quad u, v \in \mathcal{A}
$$


for some $L>0$ and some $R \geq 3\left(\sup _{u, v \in \mathcal{A}}\|u-v\|\right)$. Then, for any

$$
m>\max \left[\frac{2\{1+s(1-\alpha)\}}{1-2 \alpha}, 5\right]+1
$$

and any prescribed $\varepsilon>0$, there exists a system of ordinary differential equations

$$
\dot{x}=\mathcal{F}(x)
$$

in $\mathbb{R}^{m}$ and a bounded linear map $L: H \longrightarrow \mathbb{R}^{m}$ such that:

1. the $O D E(1.2)$ has unique solutions,

2. the restriction $\left.L\right|_{\mathcal{A}}: \mathcal{A} \longrightarrow L \mathcal{A}$ is an embedding whose image $L \mathcal{A}$ is invariant under the dynamics of (1.2),

3. for every solution $u(t)$ of (1.4) on the attractor $\mathcal{A}$ there exists a unique solution $x(t)$ of (1.2) such that

$$
u(t)=L^{-1}(x(t)),
$$

4. the $O D E$ (1.2) has a global attractor $\mathcal{X}$ that contains $L \mathcal{A}$ and is contained in the $\varepsilon$-neighbourhood of $L \mathcal{A}$, i.e. $\operatorname{dist}_{\mathrm{H}}(\mathcal{X}, L \mathcal{A}) \leq \varepsilon$.

We recall that the Hausdorff distance between two non-empty subsets $X, Y \subset H$ is defined by $\operatorname{dist}_{\mathrm{H}}(X, Y)=\max (\operatorname{dist}(X, Y)$, $\operatorname{dist}(Y, X))$.

Although item 4 is not ideal (we would like $\mathcal{X}=L \mathcal{A}$ ), we do obtain uniqueness of solutions, which is certainly desirable. This is in contrast to the construction in Eden et al. (1994), where $L \mathcal{A}$ is the global attractor, but the finite-dimensional system of ODEs obtained lacks uniqueness (in fact the finite-dimensional vector field $\mathcal{F}$ is not even continuous).

Our assumptions are strong, and it is not known whether they hold for interesting examples (i.e. partial differential equations in which the existence of an inertial manifold is not known). However, our aim here is to show that such an embedding of an attractor along with its dynamics is at least feasible under certain conditions. We hope, in particular, that our results will stimulate work on two problems of interest in themselves:

- How to bound the Assouad dimension of $\mathcal{A}-\mathcal{A}$ for a global attractor $\mathcal{A}$.

- Sharp results on the regularity of vector fields restricted to attractors.

There is no known general method for the first of these problems. For the second, using an argument due to Kukavica (2007), Pinto de Moura and Robinson (2010b) showed that $\mathcal{G}$ is 1-log-Lipschitz on the attractors of a large class of semilinear evolution equations, but this is not sufficient for our theorem. Of course, the required continuity property is trivial if $H=\mathbb{R}^{N}$ and $S(\cdot)$ is generated by a Lipschitz ordinary differential equation; 'dimension reduction' is still of interest here when $d \ll N$.

Structure of the paper. The proof of Theorem 1.2 is a blend of analytical and topological techniques and splits naturally into the following steps:

1. If $X \subset \mathbb{R}^{m}$ is a cellular set, then there is a system of ODEs in $\mathbb{R}^{m}$ that has $X$ as a global attractor consisting entirely of fixed points. As a global attractor in $\mathbb{R}^{m}$ is cellular, this provides a characterization of sets that can be global attractors of such systems of ODEs. (We follow ideas due to Günther (1995) but give a simpler proof with no piecewise linear topology.) 
2. If there is a linear embedding $\left.L\right|_{\mathcal{A}}: \mathcal{A} \longrightarrow \mathbb{R}^{m}$, then $L^{\prime} \mathcal{A}$ is a cellular subset of $\mathbb{R}^{m+1}$, where $L^{\prime}=(L, 0)$. This yields a version of Theorem 1.2 for iterated homeomorphisms assuming only that $\operatorname{dim}_{\mathrm{B}}(\mathcal{A})<\infty$.

3. When (1.3) holds, for any $\gamma>1 / 2$ there is a linear embedding with a $\gamma$-log-Lipschitz inverse (a result due to Robinson, 2010).

4. Assuming both (1.3) and (1.5), one can construct a system of ODEs in $\mathbb{R}^{m}$ that reproduces the dynamics of $\mathcal{A}$ in $L \mathcal{A}$, using the regularity of $\left(\left.L\right|_{\mathcal{A}}\right)^{-1}$ and of $\mathcal{G}$ to guarantee that it has unique solutions.

5. A suitable combination of the systems of ODEs constructed in the previous two steps produces a system that satisfies the conclusion of Theorem 1.2 .

Steps 1 and 2 rely on topological techniques; the arguments are contained in Propositions 3.1 and 2.3. whereas Lemma 2.2 and Proposition 2.4 provide the link with differential equations. Step 3 has already been dealt with in the mathematical literature and will be addressed in Section 4. where we limit ourselves to a discussion of the role played by the Assouad dimension and the embedding theorem that we will be using. The fourth step is the content of Proposition 4.2 and closes Section 4 Finally, Section 5 brings together previous results to prove Theorem 1.2.

\section{Cellularity and global attractors in $\mathbb{R}^{m}$}

This section is built on ideas from Garay (1991) and Günther (1995). The first paper singles out cellularity as a distinctive property of attractors for flows, and the second uses smoothing results from piecewise linear topology to replace general flows by flows arising from differential equations.

We begin by recalling what cellularity means. A set $C$ is called an $m$-cell if there exists a homeomorphism from $B_{\mathbb{R}^{m}}(1)$ onto $C$, where $B_{\mathbb{R}^{m}}(1)$ is the closed unit ball centred at the origin in $\mathbb{R}^{m}$. A subset $X \subseteq \mathbb{R}^{m}$ is cellular in $\mathbb{R}^{m}$ if there exists a cellular sequence for $X$, that is, a sequence $\left(C_{i}\right)_{i \in \mathbb{N}} \subseteq \mathbb{R}^{m}$ of $m$-cells that are neighbourhoods of $X$ in $\mathbb{R}^{m}$ such that $\bigcap_{i \in \mathbb{N}} C_{i}=X$. Equivalently, $X$ is cellular if given any neighbourhood $U$ of $X$ there exists an $m$-cell $C \subseteq U$ that is a neighbourhood of $X$.

Whether or not a set $X$ is cellular depends not only on its topological type, but also on how it is embedded in $\mathbb{R}^{m}$; this will be particularly relevant in Section 3 .

2.1. Global attractors for systems of ODEs are cellular sets. We begin by recalling the simple proof that global attractors of systems of ODEs are cellular. Note that the proof also shows that the global attractors of iterated homeomorphisms on $\mathbb{R}^{m}$ are cellular.

Lemma 2.1. If $X$ is the global attractor for a system of ODEs on $\mathbb{R}^{m}$, then $X$ is cellular.

Proof. Since $X$ is compact, it is contained in $B(0, R)$ for some $R>0$. Since $X$ attracts $B(0,2 R)$, there exists a $T>0$ such that $S(T) B(0,2 R) \subset B(0, R)$. Since $S(T)$ arises from a system of ODEs on $\mathbb{R}^{m}$, it is a homeomorphism; thus $\{S(k T) B(0,2 R)\}_{k=1}^{\infty}$ is a decreasing sequence of $m$-cells whose intersection is $X$.

Global attractors of semiflows on infinite-dimensional spaces are also cellular, but the proof relies on infinite-dimensional topology (Garay, 1991). It is not known whether the global attractors of semiflows on finite-dimensional spaces are cellular. 
2.2. Cellular sets are global attractors for systems of ODEs. Next we will show that if $X$ is a cellular subset of $\mathbb{R}^{m}$, then there exists a system of ordinary differential equations (2.1) with $X$ as its global attractor. Günther (1995) proved a similar result for compact sets with the shape of a finite polyhedron, but he did not need to control the size of the region of attraction (whereas we want it to be all of $\mathbb{R}^{m}$ ). By restricting ourselves to a less general setting and considering only compact sets with the shape of a point, we are able to give a simpler proof that does not involve piecewise linear topology. The difficulties arise in passing from wellknown topological results to differentiable ones. Rather than using the uniqueness of differentiable structures on $\mathbb{R}^{n}$ to do this (cf. Norton and Pugh (1991) for example) we have adopted a different approach closer to Günther (1995) in spirit.

Lemma 2.2. Given a cellular subset $X$ of $\mathbb{R}^{m}$, with $m \geq 6$, there is a $\mathcal{C}^{r}$ map $\phi: \mathbb{R}^{m} \longrightarrow[0,+\infty)$, where $r$ can be chosen to be arbitrarily large, such that

$$
\dot{x}=-\nabla \phi(x)
$$

has $X$ as a global attractor. Furthermore, the mapping $\phi$ can be chosen to satisfy:

(i) $\phi(x)=0 \Leftrightarrow x \in X$ and

(ii) $\phi$ is proper; that is, $\phi^{-1}([s, t])$ is compact for any $s<t \in \mathbb{R}$.

If Lemma 2.2 holds then $\nabla \phi(x)=0 \Leftrightarrow x \in X$ since the zeros of $\nabla \phi(x)$ are precisely the equilibria of (2.1), none of which lie outside $X$. Conversely, if $\phi: \mathbb{R}^{m+1} \rightarrow[0, \infty)$ is any $\mathcal{C}^{r}$ mapping such that $\nabla \phi(x)=0 \Leftrightarrow x \in X$ and $\phi(x)=0 \Leftrightarrow x \in X$, then by Lyapunov's theorem $X$ is a global attractor for $\dot{x}=-\nabla \phi(x)$. Thus we only need to construct such a $\phi$, which we do first on $\mathbb{R}^{m} \backslash X$ and then extend to all of $\mathbb{R}^{m}$.

The proof is a little involved because cellularity is a purely topological notion, but we want a differentiable map as an outcome. Therefore we start with the following topological result and then improve it to a differentiable one in Proposition 2.4 The set $\mathbb{S}^{m-1}$ is the unit sphere in $\mathbb{R}^{m}$, that is, $\mathbb{S}^{m-1}=\left\{x \in \mathbb{R}^{m}:\|x\|=1\right\}$.

Proposition 2.3. Let $X$ be a cellular subset of $\mathbb{R}^{m}$. There exists a homeomorphism $h: \mathbb{R}^{m} \backslash X \longrightarrow \mathbb{S}^{m-1} \times(0,+\infty)$ such that the second coordinate of $h(x)$ converges to zero when $x \longrightarrow X$.

Proof. Let $Q$ be a ball in $\mathbb{R}^{m}$ centred at the origin and large enough that $X$ is contained in the interior of $Q$. By Theorem 1 in Brown (1960) there exists a continuous map $c: Q \longrightarrow Q$ that is onto, injective on $Q \backslash X$, collapses $X$ to a single point $p$ in the interior of $Q$ and is the identity on the boundary of $Q$. It is easy to construct a homeomorphism of $Q$ onto itself that takes $p$ to 0 and is the identity on the boundary, so we can assume that $p=0$.

The properties of $c$ imply that $\left.c\right|_{Q \backslash X}: Q \backslash X \longrightarrow Q \backslash\{0\}$ is a homeomorphism and if $x \longrightarrow X$, then $c(x) \longrightarrow 0$. Extend $\left.c\right|_{Q \backslash X}$ to all of $\mathbb{R}^{m} \backslash X$ by letting it be the identity outside $Q$. Finally,

$$
h(x):=\left(\frac{c(x)}{\|c(x)\|},\|c(x)\|\right)
$$

has the required properties.

To make $h$ differentiable we require some smoothing results for manifolds, rather than maps, which we take from Kirby and Siebenmann (1977). Recall that a differential manifold is a topological manifold equipped with a differential structure, that is, an atlas of coordinate charts such that the chart changes are $\mathcal{C}^{\infty}$. A map 
between smooth manifolds is $\mathcal{C}^{\infty}$ if its local expression in charts is $\mathcal{C}^{\infty}$ and a diffeomorphism if it is invertible with a $\mathcal{C}^{\infty}$ inverse (for more detailed definitions we refer the reader to Kirby and Siebenmann, 1977).

Proposition 2.4. Let $X$ be a cellular subset of $\mathbb{R}^{m}$, with $m \geq 6$. There exists a mapping $\psi: \mathbb{R}^{m} \backslash X \longrightarrow(0,+\infty)$ of class $\mathcal{C}^{\infty}$ such that:

(i) $\nabla \psi(x) \neq 0$ for every $x \in \mathbb{R}^{m} \backslash X$,

(ii) $\psi(x) \longrightarrow 0$ when $x \longrightarrow X$, and

(iii) $\psi$ is proper.

Proof. Consider the map $h$ obtained in Proposition 2.3. We would like $\psi$ to be the second coordinate of $h$, but this choice would not be differentiable in general. Thus we first have to smooth $h$ out. Let $\Sigma$ be the differentiable structure that $\mathbb{R}^{m} \backslash X$ inherits from $\mathbb{R}^{m}$ as an open subset, and transport it via $h$ to obtain a new differentiable structure $h \Sigma$ on $\mathbb{S}^{m-1} \times(0,+\infty)$; clearly by construction $h:\left(\mathbb{R}^{m} \backslash X\right)_{\Sigma} \longrightarrow\left(\mathbb{S}^{m-1} \times(0,+\infty)\right)_{h \Sigma}$ is a diffeomorphism. Now by Kirby and Siebenmann (1977, Theorem 5.1, p. 31) (and Remark 1 following that theorem) there is a diffeomorphism $g:\left(\mathbb{S}^{m-1} \times(0,+\infty)\right)_{h \Sigma} \longrightarrow\left(\mathbb{S}^{m-1}\right)_{\sigma} \times(0,+\infty)$, where $\sigma$ is some suitable differentiable structure on $\mathbb{S}^{m-1}$ (we need the hypothesis $m>6$ precisely for this theorem to work). By Remark 1 following Kirby and Siebenmann (1977, Theorem 5.1, p. 31) one can require, and it will be technically convenient to do so, that $\operatorname{dist}(y, g(y)) \leq 1$ for every $y \in \mathbb{S}^{m-1} \times(0,+\infty)$, where dist is the maximum of the distances in $\mathbb{S}^{m-1}$ and $(0,+\infty)$.

The projection onto the second factor $\mathrm{pr}_{2}:\left(\mathbb{S}^{m-1}\right)_{\sigma} \times(0,+\infty) \longrightarrow(0,+\infty)$ is obviously a $\mathcal{C}^{\infty}$ mapping (by definition of what a product differentiable structure is) and its differential is never zero. Then define $\psi:=\operatorname{pr}_{2} \circ g \circ h$, which makes the diagram

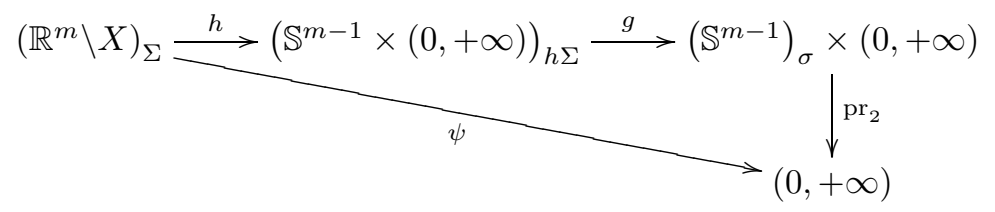

commutative. Clearly $\psi$ is $\mathcal{C}^{\infty}$, because it is a composition of $\mathcal{C}^{\infty}$ maps. Now we have to check that $\psi$ satisfies all the properties in the statement of the proposition:

( $i$ ) It is clear that $\nabla \psi(x) \neq 0$, because $g$ and $h$ are diffeomorphisms (thus their differentials are invertible) and $\operatorname{pr}_{2}$ satisfies $\nabla \operatorname{pr}_{2}(x) \neq 0$.

(iii) Let $s<t$, take a sequence $\left(x_{i}\right)_{i \in \mathbb{N}} \subseteq \psi^{-1}([s, t])$ and denote by $\left(y_{i}, z_{i}\right):=$ $g \circ h\left(x_{i}\right)$. By hypothesis $\left(\left(y_{i}, z_{i}\right)\right)_{i \in \mathbb{N}} \subseteq \mathbb{S}^{m} \times[s, t]$, which is a compact set, so the sequence $\left(\left(y_{i}, z_{i}\right)\right)_{i \in \mathbb{N}}$ must have a convergent subsequence. The pre-image of this subsequence under the homeomorphism $g \circ h$ is a convergent subsequence of $\left(x_{i}\right)_{i \in \mathbb{N}}$. This shows that $\psi^{-1}([s, t])$ is compact and $\psi$ is proper.

(ii) Let $\left(x_{i}\right)_{i \in \mathbb{N}}$ be a sequence in $\mathbb{R}^{m+1} \backslash X$ converging to $X$. We first show that $\left(\psi\left(x_{i}\right)\right)_{i \in \mathbb{N}}$ converges either to 0 or $+\infty$. If not, it has a subsequence $\left(\psi\left(x_{i_{j}}\right)\right)_{j \in \mathbb{N}}$ that is contained in a compact interval and, since $\psi$ is proper, $\left(x_{i_{j}}\right)_{j \in \mathbb{N}}$ is contained in some compact subset of $\mathbb{R}^{m} \backslash X$. This contradicts the convergence of $\left(x_{i}\right)$ to $X$.

We chose $g$ such that $\left.\operatorname{dist}\left(g \circ h\left(x_{i}\right)\right), h\left(x_{i}\right)\right)<1$ and defined dist as the maximum of the distances in $\mathbb{S}^{m-1}$ and $(0,+\infty)$, which implies that

$$
\operatorname{dist}\left(\psi\left(x_{i}\right), \mathrm{pr}_{2} \circ h\left(x_{i}\right)\right)=\operatorname{dist}\left(\operatorname{pr}_{2} \circ g \circ h\left(x_{i}\right), \operatorname{pr}_{2} \circ h\left(x_{i}\right)\right)<1
$$


as well. Since $\psi\left(x_{i}\right)$ converges to either 0 or $+\infty$ and $\operatorname{pr}_{2} \circ h\left(x_{i}\right) \longrightarrow 0$ as stated in Proposition 2.3, it follows that $\psi\left(x_{i}\right) \longrightarrow 0$.

Proof of Lemma 2.2. We will construct inductively a sequence of maps $\psi_{k}$, each $\psi_{k}$ of class $\mathcal{C}^{k}$, such that $\phi:=\psi_{k}$ satisfies the lemma for $r=k$. As a first step extend the mapping $\psi$ given by Proposition 2.4 to all of $\mathbb{R}^{m}$ by letting it assume the value 0 on $X$, and call it $\psi_{0}$. This $\psi_{0}$ is continuous but not differentiable near $X$, and we now use an argument hinted at by Günther (1995) to improve $\psi_{0}$ to $\psi_{1}$.

The idea is to let $\psi_{1}:=b \circ \psi_{0}$, where $b:[0,+\infty) \longrightarrow[0,+\infty)$ is some diffeomorphism of class $\mathcal{C}^{1}$ whose derivative near 0 is sufficiently small to overcome the "roughness" of $\psi_{0}$ near $X$. Formally, for $x \in \mathbb{R}^{m} \backslash X$,

$$
\frac{\partial}{\partial x_{i}}\left(b \circ \psi_{0}\right)(x)=\left(b^{\prime} \circ \psi_{0}\right)(x) \frac{\partial \psi_{0}}{\partial x_{i}}(x)
$$

and as $x \longrightarrow X$ (and consequently $t=\psi_{0}(x) \longrightarrow 0$ ) we need $b^{\prime}(t)$ to converge to 0 faster than $\frac{\partial \psi_{0}}{\partial x_{i}}(x)$ grows. We now show how to find such a $b$.

For any $t \in(0,+\infty)$ let $F_{t}:=\left\{x \in \mathbb{R}^{m} \backslash X: \psi_{0}(x)=t\right\}$ and

$$
M(t):=\max _{x \in F_{t}}\left\{\left|\frac{\partial \psi_{0}}{\partial x_{1}}(x)\right|, \ldots,\left|\frac{\partial \psi_{0}}{\partial x_{m-1}}(x)\right|\right\} .
$$

Since each $F_{t}$ is compact, because $\psi_{0}$ is proper, $M(t)$ is well defined. The condition $\nabla \psi_{0}(x) \neq 0$ for $x \in \mathbb{R}^{m} \backslash X$ implies $M(t)>0$ for every $t>0$, and clearly by construction $M\left(\psi_{0}(x)\right) \geq\left|\frac{\partial \psi_{0}}{\partial x_{i}}(x)\right|$ for each $x \in \mathbb{R}^{m} \backslash X$ and $1 \leq i \leq m$. Suppose for a moment that we find a diffeomorphism $b$ such that $b^{\prime}(t) \leq \frac{t}{M(t)}$ for every $t>0$. Then we have, for any $1 \leq i \leq m$,

$$
\frac{\partial}{\partial x_{i}}\left(b \circ \psi_{0}\right)(x)=\left(b^{\prime} \circ \psi_{0}\right)(x) \frac{\partial \psi_{0}}{\partial x_{i}}(x) \leq \frac{\psi_{0}(x)}{M\left(\psi_{0}(x)\right)} \frac{\partial \psi_{0}}{\partial x_{i}}(x) \leq \psi_{0}(x),
$$

which goes to 0 as $x \longrightarrow X$. Hence $\psi_{1}:=b \circ \psi_{0}$ is $\mathcal{C}^{1}$ on $\mathbb{R}^{m}$, and its gradient on $X$ is zero. It is still clearly regular on $\mathbb{R}^{m} \backslash X$ and goes to zero as $x \longrightarrow X$, so $\phi:=\psi_{1}$ satisfies the lemma for $r=1$.

If $t / M(t)$ were continuous, one could simply take $b(t)$ to be the primitive of $t / M(t)$ with $b(0)=0$. One could do this using Morse theory, but we adopt a more elementary approach, first showing that $M(t)$ is upper semicontinuous, i.e. that for each $s \in \mathbb{R}$, the set $\{t \in(0,+\infty): M(t)<s\}$ is open.

To this end, fix $t_{0} \in \mathbb{R}$ and $s \in \mathbb{R}$ such that $M\left(t_{0}\right)<s$. We have to prove that for $t$ close enough to $t_{0}, M(t)<s$. At each point $x \in F_{t_{0}}$ one has $\left|\frac{\partial \psi_{0}}{\partial x_{i}}(x)\right|<s$ for all $1 \leq i \leq m$, so, by continuity, there exists a neighbourhood $U_{x}$ of $x$ in $\mathbb{R}^{m} \backslash X$ such that $\left|\frac{\partial \psi_{0}}{\partial x_{i}}(y)\right|<s$ for all $y \in U_{x}$ and $1 \leq i \leq m$. The set $U:=\bigcup_{x \in F_{t_{0}}} U_{x}$ is a neighbourhood of $F_{t_{0}}$ in $\mathbb{R}^{m} \backslash X$. Now clearly $F_{t_{0}}=\bigcap_{\varepsilon>0} F_{\left[t_{0}-\varepsilon, t_{0}+\varepsilon\right]}$, where $F_{\left[t_{0}-\varepsilon, t_{0}+\varepsilon\right]}:=\left\{y \in \mathbb{R}^{m} \backslash X: \psi_{0}(y) \in\left[t_{0}-\varepsilon, t_{0}+\varepsilon\right]\right\}$. Again because $\psi_{0}$ is proper, each $F_{\left[t_{0}-\varepsilon, t_{0}+\varepsilon\right]}$ is compact, so there exists $\varepsilon>0$ such that $F_{\left[t_{0}-\varepsilon, t_{0}+\varepsilon\right]} \subseteq U$. But then for $t \in\left[t_{0}-\varepsilon, t_{0}+\varepsilon\right]$ we have $M\left(t_{0}\right)<s$, as required.

We can now find $b$. Since $M(t)$ is upper semicontinuous, so is $M(t) / t$, and consequently $t / M(t)$ is lower semicontinuous. By a classical insertion theorem (Dowker, 1951, Theorem 4, p. 222) it follows that there exists a continuous mapping $0<c(t)<t / M(t)$. Taking for $b(t)$ the primitive of $c(t)$ with $b(0)=0$ we are finished. 
This argument can easily be adapted to provide the inductive step in the construction of $\psi_{k+1}$ from $\psi_{k}$. We again let $\psi_{k+1}:=b \circ \psi_{k}$ for a suitable $\mathcal{C}^{k+1}$ diffeomorphism $b:[0,+\infty) \longrightarrow[0,+\infty)$, but now there are conditions to be placed on the rate at which $b^{(l)}(t) \longrightarrow 0$ as $t \longrightarrow 0$ for every $0 \leq l \leq k+1$. Indeed, for any multi-index $\alpha$ with $|\alpha|=k+1$ we have

$$
\frac{\partial^{\alpha} \psi_{k+1}}{\partial x^{\alpha}}=b^{\prime} \circ \psi_{k} \frac{\partial^{\alpha} \psi_{k}}{\partial x^{\alpha}}+P\left(\frac{\partial^{\beta} \psi_{k}}{\partial x^{\beta}}, b^{(l)}\right)
$$

on $\mathbb{R}^{m} \backslash X$, where $P$ is a polynomial in partial derivatives of $\psi_{k}$ of order $\leq k$ and derivatives of $b$ of order $l \leq k+1$. Hence we now need to choose $b$ subject to the conditions $b^{(l)}(0)=0$ for every $l \leq k+1$ and $b^{\prime} \circ \psi_{k}(x) \frac{\partial^{\alpha} \psi_{k}}{\partial x^{\alpha}}(x) \longrightarrow 0$ for $|\alpha|=k+1$ and $x \longrightarrow X$. The first one is easy to achieve; for the second one, just reread the proof from the beginning letting

$$
M(t):=\max _{\substack{x \in F_{t} \\|\alpha|=k+1}}\left\{\left|\frac{\partial^{\alpha} \psi_{k}}{\partial x^{\alpha}}(x)\right|\right\} .
$$

Combining these results we obtain a characterization of those sets that can be the global attractors of systems of ordinary differential equations in finite-dimensional spaces. The restriction $m \geq 6$ can be eliminated; see Norton and Pugh (1991).

Corollary 2.5. A compact subset $X$ of $\mathbb{R}^{m}(m \geq 6)$ is the global attractor of some system of ordinary differential equations on $\mathbb{R}^{m}$ if and only if it is cellular.

\section{Finite-Dimensional attractors as ATtractors}

\section{IN FINITE-DIMENSIONAL SYSTEMS}

In this section we show that if $\mathcal{A}$ is a finite-dimensional attractor in a Hilbert space $H$, then there is a linear embedding $L^{\prime}: H \rightarrow \mathbb{R}^{m+1}$ (for some $m$ ) such that $L^{\prime} \mathcal{A}$ is a cellular subset of $\mathbb{R}^{m+1}$; it follows that $L^{\prime} \mathcal{A}$ can be made the attractor of some finite-dimensional system of ODEs.

Any set $\mathcal{A} \subset H$ with finite box-counting dimension can be embedded into $\mathbb{R}^{m}$ using a linear map $L$ : if $\operatorname{dim}_{\mathrm{B}}(\mathcal{A})=d$, then one can take any $m>2 d+1$. This result, originally due to Mañé (1981), was improved to guarantee that $\left.L^{-1}\right|_{L \mathcal{A}}$ is Hölder by Foias and Olson (1996) and then further refined by Hunt and Kaloshin (1999), who provided explicit bounds on the Hölder exponent. (We will discuss the existence of more 'regular' embeddings in the next section.) The assumption that such an embedding exists is therefore satisfied for any such 'finite-dimensional' attractor.

Proposition 3.1. Let $\mathcal{A}$ be a global attractor in $H$ and let $L: H \longrightarrow \mathbb{R}^{m}$ be a linear embedding. Then the map $L^{\prime}: H \longrightarrow \mathbb{R}^{m+1}$ defined by $L^{\prime} u=(L u, 0)$ is a linear embedding whose image $L^{\prime} \mathcal{A}$ is cellular in $\mathbb{R}^{m+1}$, provided $m \geq 3$.

Due to the fact mentioned above that the cellularity of a set depends on how it is embedded, we cannot prove Proposition 3.1 directly by saying that $\mathcal{A}$ is cellular (because it is an attractor) and then $L \mathcal{A}$ is cellular because it is homeomorphic, via $L$, to $\mathcal{A}$. We need to use a different property of $\mathcal{A}$, which is invariant under homeomorphisms. This is shape. Shape theory is a weakening of homotopy theory that makes it extremely useful to deal with complicated sets, roughly by overlooking their local structure. The advantage for us is that if two spaces are homeomorphic, then they have the same shape. In fact, something even stronger is true: if two 
spaces have the same homotopy type, then they have the same shape. We refer the reader to Borsuk (1975) and Mardešić and Segal (1982) for detailed information about shape theory, which is a powerful tool in the study of topological dynamics (see Günther and Segal (1993), Sanjurjo (1995), Robinson (1999)).

Proof of Proposition 3.1. By Theorem 3.6 in Kapitanski and Rodnianski (2000, p. 233) the set $\mathcal{A}$ has the same shape as $H$. It is a standard fact that $H$ has the homotopy type of a point, because the map $H \times[0,1] \ni(u, t) \longrightarrow(1-t) \cdot u \in H$ provides a homotopy between the identity id $: H \longrightarrow H$ and the constant map $0: H \longrightarrow H$. Therefore $H$ has the shape of a point and consequently so does $\mathcal{A}$. Since shape is invariant under homeomorphisms, $L \mathcal{A}$ also has the shape of a point. Thu 1 by Daverman (1986, Corollary 5A, Section 18) the set $L \mathcal{A} \times\{0\}$ is cellular in $\mathbb{R}^{m+1}$ provided $m \geq 3$. But $L \mathcal{A} \times\{0\}$ is precisely $L^{\prime} \mathcal{A}$.

Given that in our final result $L \mathcal{A}$ is not the global attractor but only closely approximated by global attractors $\mathcal{X}$, one may wonder if the requirement of cellularity is due only to our method of proof. It is not: Given an open neighbourhood $U$ of $L \mathcal{A}$, find a system of ODEs that has a global attractor $\mathcal{X} \subseteq U$. Since $L \mathcal{A}$ is invariant and $\mathcal{X}$ is a global attractor, $L \mathcal{A} \subseteq \mathcal{X}$. The set $\mathcal{X}$ is cellular, so there exists a cell $C \subseteq U$ that is a neighbourhood of $\mathcal{X}$, hence of $L \mathcal{A}$; thus $L \mathcal{A}$ is cellular.

An interesting consequence of Proposition 3.1 is the following, which is essentially a discrete version of the main theorem of this paper. The proof is adapted from Robinson (1999); enhancing $L$ to $L^{\prime}=(L, 0)$ fixes a gap in the argument there.

Proposition 3.2. Let $\mathcal{A}$ be the global attractor of a map $S: H \rightarrow H$ such that $\left.S\right|_{\mathcal{A}}$ is a homeomorphism and $\operatorname{dim}_{\mathrm{B}}(\mathcal{A})=d$. Then for any $k>\max (2 d+4,5)$ and $\varepsilon>0$ there exist homeomorphisms $L: \mathcal{A} \rightarrow \mathbb{R}^{k}$ and $f: \mathbb{R}^{k} \rightarrow \mathbb{R}^{k}$ such that

- $\left.f\right|_{L \mathcal{A}}$ reproduces the dynamics on $\mathcal{A}$, i.e. $\left.f\right|_{L \mathcal{A}}=L \circ S \circ L^{-1}$, and

- the dynamical system on $\mathbb{R}^{k}$ generated by $f$ has an attractor $\mathcal{X}$ with

$$
L \mathcal{A} \subseteq \mathcal{X} \subseteq B_{\varepsilon}(L \mathcal{A})
$$

where $B_{\varepsilon}(L \mathcal{A})$ denotes the $\varepsilon$-neighbourhood of $L \mathcal{A}$ in $\mathbb{R}^{k}$.

Proof. First choose any $m>2 d+1$ and a linear embedding $L^{\prime}$ of $\mathcal{A}$ into $\mathbb{R}^{m}$; then set $L=\left(L^{\prime}, 0,0,0\right)$ and let $k=\max (m+3,6)$. Define $h^{\prime}: L \mathcal{A} \rightarrow L \mathcal{A}$ by $h^{\prime}(x)=L S\left(L^{-1} x\right)$. Since $L \mathcal{A}$ is compact, $\left|h^{\prime}(x)-x\right| \leq \delta:=\operatorname{diam}(L \mathcal{A})$ for every $x \in L \mathcal{A}$. Since $L \mathcal{A}$ is a $Z$-set, one can use an extension theorem due to Geoghegan and Summerhill (1973) to extend $h^{\prime}$ to a homeomorphism $h: \mathbb{R}^{k} \rightarrow \mathbb{R}^{k}$ such that $|h(x)-x| \leq \delta$ for all $x \in \mathbb{R}^{k}$ and $h(x)=x$ for all $x$ outside $B_{\delta}(L \mathcal{A})$. Note that $B_{2 \delta}(L \mathcal{A})$ is positively invariant under $h$. Reduce $\varepsilon$ if necessary so that $\varepsilon<2 \delta$.

Now consider the ODE (2.1) on $\mathbb{R}^{k}$ whose existence is guaranteed by Lemma 2.2 since $L \mathcal{A}$ is cellular. Since $L \mathcal{A}$ is the global attractor of this equation, there exists a $T>0$ such that $B_{2 \delta}(L \mathcal{A}) \cdot t \subset B_{\varepsilon}(L \mathcal{A})$ for all $t \geq T$. The homeomorphism $f$, defined by composing $h$ with the time $T$ map of the flow induced by (2.1), satisfies the required properties.

Note that this result only requires $\mathcal{A}$ to have finite box-counting dimension, unlike Theorem 1.2. which requires the much stronger assumption that $\operatorname{dim}_{\mathrm{A}}(\mathcal{A}-\mathcal{A})<\infty$.

\footnotetext{
${ }^{1}$ Daverman uses the concept of cell-likeness instead of "having the shape of a point", but both are equivalent. See Section 15 in Daverman (1986).
} 


\section{Embedding the dynamics on $\mathcal{A}$ into Euclidean space}

We now consider the problem of embedding a set $\mathcal{A}$ into $\mathbb{R}^{m}$ in such a way that (1.2) can reproduce the dynamics on $\mathcal{A}$. We therefore require the right-hand side $f(x)$ of (1.2) to bear a close relation with $\mathcal{G}$ on the image $L \mathcal{A}$ of $\mathcal{A}$ : essentially it needs to be $L \mathcal{G} L^{-1}$. To guarantee uniqueness of solutions for (1.2) some regularity has to be required of $L \mathcal{G} L^{-1}$; the standard one is Lipschitz continuity, but this can in fact be weakened to $1-\log$-Lipschitz continuity. Since $\mathcal{G}$ was already assumed to be $\alpha$-log-Lipschitz continuous, only $L$ and $L^{-1}$ need to be taken care of.

Many current embedding results provide linear maps $L: H \rightarrow \mathbb{R}^{m}$ that are embeddings (see Robinson, 2009, for example); such maps are Lipschitz, so the main issue is the regularity of $L^{-1}$. Suppose for a moment that $L^{-1}$ was also required to be Lipschitz restricted to $L \mathcal{A}$, so that $L$ would be bi-Lipschitz. That is, there would exist a constant $C>0$ such that

$$
\frac{1}{C}\|u-v\| \leq|L(u)-L(v)| \leq C\|u-v\| \quad \text { for all } \quad u, v \in \mathcal{A},
$$

where $|\cdot|$ denotes some norm in $\mathbb{R}^{m}$. Assouad (1983) introduced a dimension, the Assouad dimension $\operatorname{dim}_{\mathrm{A}}$ (whose definition is recalled below), which is invariant under bi-Lipschitz mappings and is finite for subsets of Euclidean space. Thus if $\mathcal{A}$ is to be embedded in a bi-Lipschitz way into $\mathbb{R}^{m}$, we must have $\operatorname{dim}_{\mathrm{A}}(\mathcal{A})<\infty$.

A metric space $(X, d)$ is said to be $(M, s)$-homogeneous (or simply homogeneous) if any ball of radius $r$ can be covered by at most $M(r / \rho)^{s}$ smaller balls of radius $\rho$, for some $M \geq 1$ and $s \geq 0$. The Assouad dimension of $X, \operatorname{dim}_{\mathrm{A}}(X)$, is the infimum of all $s$ such that $(X, \bar{d})$ is $(M, s)$-homogeneous, for some $M \geq 1$ (of course, if $X$

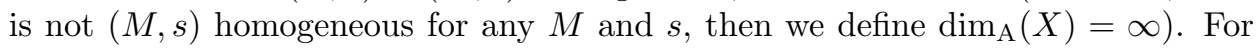
more details, see Luukkainen (1998) and Olson (2002).

Olson and Robinson (2010) proved that if $\operatorname{dim}_{\mathrm{A}}(\mathcal{A}-\mathcal{A})<\infty$, then there exists a bi-log-Lipschitz embedding of $\mathcal{A}$ into a Euclidean space; a version of this result with a better logarithmic exponent was subsequently proved by Robinson (2010).

Theorem 4.1 (Robinson, 2010). Let $\mathcal{A}$ be a compact subset of a real Hilbert space $H$ such that $\operatorname{dim}_{\mathrm{A}}(\mathcal{A}-\mathcal{A})<s<m$. If

$$
\gamma>\frac{2+m}{2(m-s)}
$$

then there exists a prevalent set 2 of linear maps $L: H \longrightarrow \mathbb{R}^{m}$ that are injective on $X$ and $\gamma$-almost bi-Lipschitz; i.e. there exist $\delta_{L}>0, C_{L}>0$ such that

$$
\frac{1}{C_{L}} \frac{\|u-v\|}{(-\log \|u-v\|)^{\gamma}} \leq|L(u)-L(v)| \leq C_{L}\|u-v\|,
$$

for all $u, v \in \mathcal{A}$ with $\|u-v\| \leq \delta_{L}$.

Note that for any $\gamma>1 / 2$ we can choose $m$ large enough to obtain a $\gamma$-almost biLipschitz embedding into $\mathbb{R}^{m}$. Pinto de Moura and Robinson (2010a) showed that this bound on the logarithmic exponent $\gamma$ in Theorem 4.1 is sharp as $m \longrightarrow \infty$.

\footnotetext{
${ }^{2}$ The term 'prevalence' was coined by Hunt et al. (1992) and generalizes the notion of 'Lebesgue almost every' from finite to infinite-dimensional spaces. The same notion was, essentially, used earlier by Christensen (1973) in a study of the differentiability of Lipschitz mappings between infinite-dimensional spaces. Let $V$ be a normed linear space. A Borel subset $S \subset V$ is prevalent if there exists a compactly supported probability measure $\mu$ such that $\mu(S+x)=1$, for all $x \in V$. In particular, if $S$ is prevalent, then $S$ is dense in $V$.
} 
Although reasonable, the hypothesis $\operatorname{dim}_{\mathrm{A}}(\mathcal{A}-\mathcal{A})<\infty$ is extremely restrictive, since there are currently no methods available to bound the Assouad dimension of global attractors. Even then, $\operatorname{dim}_{\mathrm{A}}(\mathcal{A})<\infty$ still does not imply that $\operatorname{dim}_{\mathrm{A}}(\mathcal{A}-$ $\mathcal{A})<\infty$ (see Olson (2002)). The only attractors known to satisfy this condition are those that are subsets of inertial manifolds; but, of course, in this case, a finitedimensional system of ODEs that reproduces the behaviour on $\mathcal{A}$ is already known to exist, making this class of examples of little interest. Nevertheless, we will assume that $\operatorname{dim}_{\mathrm{A}}(\mathcal{A}-\mathcal{A})<\infty$ in order to study its consequences.

To conclude this section we use Theorem 4.1 above to construct a system of ordinary differential equations with unique solutions that reproduces the dynamics on $\mathcal{A}$ under the assumptions of Theorem 1.2 .

Proposition 4.2. Under the hypotheses of Theorem 1.2 and with the same notation, for any

$$
m>\frac{2\{1+s(1-\alpha)\}}{1-2 \alpha}
$$

there exist a system of ODEs in $\mathbb{R}^{m}$,

$$
\dot{x}=g(x),
$$

and a bounded linear map $L: H \longrightarrow \mathbb{R}^{m}$ such that:

1. the function $g: \mathbb{R}^{m} \longrightarrow \mathbb{R}^{m}$ is bounded and Lipschitz except for a logarithmic correction,

2. the ODE (4.4) has unique solutions,

3. the restriction $\left.L\right|_{\mathcal{A}}: \mathcal{A} \longrightarrow L \mathcal{A}$ is an embedding whose image is invariant under (4.4),

4. for every solution $u(t)$ of (1.4) on the attractor $\mathcal{A}$ there exists a unique solution $x(t)$ of (4.4) such that

$$
u(t)=L^{-1}(x(t)) .
$$

Proof. It follows from Theorem 4.1 that there exists a bounded linear map $L$ from $H$ into $\mathbb{R}^{m}$ that is injective on $\mathcal{A}$ and has a Lipschitz continuous inverse on $L \mathcal{A}$ except for a logarithmic correction term with logarithmic exponent $\gamma$.

If $x(t)=L u(t)$, where $u(t) \in \mathcal{A}$, then the embedded vector field on $L \mathcal{A}$ is given by $\dot{x}=g_{1}(x):=L \mathcal{G} L^{-1}(x)$ for $x \in L \mathcal{A}$. The function $g_{1}: L \mathcal{A} \longrightarrow \mathbb{R}^{m}$ is certainly continuous and bounded, since $L \mathcal{A}$ is compact; it is also log-Lipschitz.

Indeed, given $u, v \in H$, define $L u=x$ and $L v=y$. It follows from Theorem 4.1 that

$$
|x-y| \geq \frac{1}{C_{L}} \frac{\left\|L^{-1} x-L^{-1} y\right\|}{\left(-\log \left(\left\|L^{-1} x-L^{-1} y\right\|\right)\right)^{\gamma}},
$$

where we increase $C_{L}$ if necessary so that $C_{L}>4 \max _{x, y \in L \mathcal{A}}|x-y|$. Consequently, since $|L u-L v| \leq C_{L}\|u-v\|$, for every $x, y \in L \mathcal{A}$,

$$
\begin{aligned}
\left\|L^{-1} x-L^{-1} y\right\| & \leq C_{L}\left(-\log \left(\left\|L^{-1} x-L^{-1} y\right\|\right)\right)^{\gamma}|x-y| \\
& \leq C_{L}\left(\log \left(\frac{C_{L}}{|x-y|}\right)\right)^{\gamma}|x-y| .
\end{aligned}
$$


Since we assumed that $\mathcal{G}$ is $\alpha$-log-Lipschitz continuous, it follows that

$$
\left|g_{1}(x)-g_{1}(y)\right| \leq\|L\|_{\mathrm{op}} C_{\mathcal{G}} C_{L}|x-y|\left(\log \frac{M}{|x-y|}\right)^{\alpha+\gamma}=: \omega(|x-y|)
$$

with $M=\max \left(C_{L}, R\right)$. Hence $g_{1}$ is $(\alpha+\gamma)$-log-Lipschitz continuous. The modulus of continuity $\omega$ of $g_{1}$ is a convex continuous function; one can now use the extension theorem due to McShane (1934) (see also Stein, 1982) to extend the function $g_{1}$ to a function $g: \mathbb{R}^{m} \longrightarrow \mathbb{R}^{m}$ that has essentially the same modulus of continuity,

$$
|g(x)-g(y)| \leq C \omega(|x-y|),
$$

for some $C>0$. It follows from (4.6) that there exists a $T>0$ such that the initial value problem

$$
\frac{\mathrm{d} x}{\mathrm{~d} t}=g(x), \quad x(0)=x_{0}
$$

has at least one solution on $[0, T]$.

Since the modulus of continuity $\omega(r)$ of $g$ is continuous for $r \geq 0$, convex and satisfies

$$
\int_{0}^{1} \frac{\mathrm{d} r}{\omega(r)} \propto \int_{\ln M}^{\infty} s^{-(\alpha+\gamma)} \mathrm{d} s=+\infty
$$

provided that $\alpha+\gamma \leq 1$, we can use Osgood's Criterion (see Hartman (1964), for example) to show that (4.7) has at most one solution on any interval $[0, T]$. Since $g$ is continuous and bounded from $\mathbb{R}^{m}$ into $\mathbb{R}^{m}$, it follows that any solution of the initial value problem (4.7) exists for all time. Therefore the solution of (4.7) through $x_{0}=L u_{0}$ with $u_{0} \in \mathcal{A}$ can be uniquely given by

$$
x(t)=L u(t) .
$$

Finally, observe that requiring $\alpha+\gamma \leq 1$ yields condition (4.3) on using (4.1).

\section{Making $L \mathcal{A}$ an ATtractor}

In the previous section we embedded $\mathcal{A}$ into some finite-dimensional space $\mathbb{R}^{m}$ via a linear map $L: H \longrightarrow \mathbb{R}^{m}$ and showed that there is a differential equation (4.4) in $\mathbb{R}^{m}$ that has unique solutions and reproduces the dynamics of $\mathcal{A}$ on $L \mathcal{A}$. To obtain a complete translation of the situation in $H$ onto $\mathbb{R}^{m}$ we would like $L \mathcal{A}$ to be a global attractor for (4.4), which is not usually the case. As we mentioned in the Introduction, we will only be able to modify (4.4) in such a way that the new dynamical system still reproduces the dynamics of $\mathcal{A}$ on $L \mathcal{A}$ and has a global attractor $\mathcal{X}$ lying within any prescribed (arbitrarily small) neighbourhood of $L \mathcal{A}$. We do not know if one can construct a vector field such that $L \mathcal{A}$ itself, with the dynamics projected from $\mathcal{A}$, is a global attractor.

In this final section we assemble all our previous results to obtain a system of ODEs (1.2) that reproduces on $L \mathcal{A}$ the dynamics on $\mathcal{A}$ and has a global attractor $\mathcal{X}$ as close to $L \mathcal{A}$ as required.

Proof of Theorem 1.2. Use Proposition 3.1 to replace the mapping $L$ obtained in Proposition 4.2 by a new one $L^{\prime}: H \longrightarrow \mathbb{R}^{m+1}$ with the additional property that its image is cellular. To keep notation simple we rename $L^{\prime}$ as $L$ and $m+1$ as $m$.

Use Lemma 2.2 to obtain a $\mathcal{C}^{r}$ mapping $\phi: \mathbb{R}^{m} \longrightarrow[0,+\infty)$ such that $L \mathcal{A}$ is a global attractor for $\dot{x}=-\nabla \phi$. Since $\phi$ is proper, there exists $\delta>0$ such that 
$P:=\left\{x \in \mathbb{R}^{m}: \phi(x) \leq \delta\right\} \subseteq B_{\varepsilon}(L \mathcal{A})$. Finally, let $\theta: \mathbb{R}^{m} \longrightarrow[0,1]$ be a $\mathcal{C}^{\infty}$ cutoff function such that $\theta \equiv 1$ on $L \mathcal{A}$ and $\theta \equiv 0$ outside of $P$. Take the mapping $g$ obtained in Proposition 4.2 and multiply it by $\theta$ to make it zero outside of $P$. We shall define $f:=\theta g$; clearly $\dot{x}=f(x)$ still reproduces the dynamics of $\mathcal{A}$ on $L \mathcal{A}$.

Now consider equations (2.1) and (5.1):

$$
\begin{aligned}
& \dot{x}=-\nabla \phi(x), \\
& \dot{x}=f(x)-\nabla \phi(x) .
\end{aligned}
$$

Observe that the right-hand sides of (2.1) and (5.1) coincide for $x \notin P$. Therefore, since $\mathbb{R}^{m} \backslash P$ is negatively invariant for (2.1), it is also negatively invariant for (5.1) and it follows that $P$ is positively invariant for (5.1).

The sets $\overline{P \cdot[t,+\infty)}$ are compact (being closed subsets of $P$ ) and decreasing with increasing $t$. It is standard that

$$
\mathcal{X}:=\bigcap_{t \geq 0} \overline{P \cdot[t,+\infty)}
$$

is invariant and attracts $P$; i.e., given any $\delta>0$ there exists $T_{\delta}>0$ such that $P \cdot\left[T_{\delta},+\infty\right) \subseteq B_{\delta}(\mathcal{X})$ (see Ladyzhenskaya, 1991, Theorem 2.1). By construction, $\mathcal{X}$ is contained in $B_{\varepsilon}(L \mathcal{A})$.

(1) $\mathcal{X}$ is a global attractor. Fix a bounded set $B \subseteq \mathbb{R}^{m}$ and let

$$
C:=\sup _{x \in B} \phi(x) \text { and } c:=\inf _{x \in B-P}\|\nabla \phi\|^{2} .
$$

Observe that $c>0$ because $\nabla \phi$ only vanishes on $L \mathcal{A}$, of which $P$ is a neighbourhood. Thus there exists $T>0$ large enough so that $C-c T<\delta$ holds.

We now claim that $x \cdot[T,+\infty) \subseteq P$ for any $x \in B$. Since $P$ is positively invariant it clearly suffices to show that $x \cdot t \in P$ for some $t \in[0, T]$. We reason by contradiction, so assume that $x \cdot[0, T] \subseteq \mathbb{R}^{m} \backslash P$. By the mean value theorem

$$
\phi(x \cdot T)=\phi(x)+\left.\frac{\mathrm{d}}{\mathrm{d} s} \phi(x \cdot s)\right|_{s=\xi} T
$$

for some $\xi \in[0, T]$. Now

$$
\left.\frac{\mathrm{d}}{\mathrm{d} s} \phi(x \cdot s)\right|_{s=\xi}=\langle\nabla \phi(x \cdot \xi), \dot{x}(\xi)\rangle=-\|\nabla \phi(x \cdot \xi)\|^{2} \leq-c,
$$

where we have used the fact that $\dot{x}(\xi)=-\nabla \phi(x \cdot \xi)$ because $x \cdot \xi \notin P$ by assumption and $-\|\nabla \phi(x \cdot \xi)\|^{2} \leq-c$ by the same token. With the above equation and the fact that $\phi(x) \leq C$ because $x \in P$,

$$
\phi(x \cdot T) \leq C-c T<\delta,
$$

which is a contradiction since then $x \cdot T \in P$ by definition.

Thus we see that $B \cdot[T,+\infty) \subseteq P$. Since given any $\delta>0$ there exists $T_{\delta}>0$ such that $P \cdot\left[T_{\delta},+\infty\right) \subseteq B_{\delta}(\mathcal{X})$, it follows that $B \cdot\left[T+T_{\delta},+\infty\right) \subseteq P \cdot\left[T_{\delta},+\infty\right) \subseteq B_{\delta}(\mathcal{X})$. Thus for $t \geq T+T_{\delta}$ one has $\operatorname{dist}(B \cdot t, \mathcal{X})<\delta$. This implies that $\operatorname{dist}(B \cdot t, \mathcal{X}) \longrightarrow 0$ as $t \longrightarrow+\infty$.

(2) $\mathcal{X}$ contains $L \mathcal{A}$. Since $\nabla \phi$ vanishes on $L \mathcal{A}$ and $\theta \equiv 1$ on it, (5.1) reduces to $\dot{x}=g(x)$ when $x \in L \mathcal{A}$. Thus $L \mathcal{A}$ is invariant for (5.1), and it is an immediate consequence of the fact that $L \mathcal{A} \subseteq P$ and the expression for $\mathcal{X}$ that $L \mathcal{A} \subseteq \mathcal{X}$ (alternatively, since $\mathcal{X}$ is the maximal compact invariant set in $\mathbb{R}^{m}$, clearly $L \mathcal{A} \subseteq$ $\mathcal{X})$. 


\section{Conclusion}

In this paper, we have shown that if the compact set $\mathcal{A} \subset H$ is the global attractor associated with a dissipative evolution equation in $H$ such that the vector field $\mathcal{G}$ is $\alpha$-log-Lipschitz continuous on $\mathcal{A}(\alpha<1 / 2)$ and $\operatorname{dim}_{\mathrm{A}}(\mathcal{A}-\mathcal{A})=d$, then there is an ordinary differential equation in some $\mathbb{R}^{k}$ that has unique solutions and reproduces the dynamics on $\mathcal{A}$. Moreover, we proved that the dynamical system generated by this new ordinary differential equation has a global attractor $\mathcal{X}$ arbitrarily close to $L \mathcal{A}$, where $L$ is a bounded linear map from $H$ into $\mathbb{R}^{k}$ that is injective on $\mathcal{A}$. Even under the strong assumptions that we have imposed it remains an interesting open question whether there is a similar construction for which $\mathcal{X}$ is precisely $L \mathcal{A}$.

The results presented in this paper highlight the importance of finding a general method to bound the Assouad dimension of the set $\mathcal{A}-\mathcal{A}$, where $\mathcal{A}$ is a global attractor associated with a partial differential equation in $H$. In addition, the assumption that the vector field $\mathcal{G}$ is $\alpha$-log Lipschitz continuous on the global attractor $\mathcal{A}$, with $\alpha<1 / 2$, appears to be too strong in the light of current results, and it would be of great interest to refine these further.

\section{REFERENCES}

1. P. Assouad. Plongements Lipschitziens dans $\mathbb{R}^{N}$. Bull. Soc. Math. France, 111:429-448, 1983. MR763553 (86f:54050)

2. J.E. Billotti and J.P. LaSalle. Dissipative periodic processes. Bull. Amer. Math. Soc. 77:10821088, 1971. MR0284682 (44:1906)

3. K. Borsuk. Theory of Shape, volume 59 of Monografie Matematyczne. Polish Scientific Publishers, Warszawa, 1975. MR0418088 (54:6132)

4. M. Brown. A proof of the generalized Schoenflies theorem. Bull. Amer. Math. Soc., 66:74-76, 1960. MR0117695 (22:8470b)

5. V.V. Chepyzhov and M.I. Vishik. Attractors for equations of mathematical physics, volume 49 of American Mathematical Society Colloquium Publications. American Mathematical Society, 2002. MR1868930 (2003f:37001c)

6. J.P.R. Christensen. Measure theoretic zero sets in infinite dimensional spaces and applications to differentiability of Lipschitz mappings. Publ. Dép. Math. (Lyon), 10(2):29-39, 1973. MR0361770 (50:14215)

7. P. Constantin and C. Foias. Navier-Stokes Equations. University of Chicago Press, Chicago, 1988. MR 972259 (90b:35190)

8. P. Constantin, C. Foias, B. Nicolaenko and R. Temam. Integral manifolds and inertial manifolds for dissipative partial differential equations. Springer-Verlag, New York, 1989. MR966192 (90a:35026)

9. R.J. Daverman. Decomposition of manifolds. Academic Press Inc., London, 1986. MR 872468 (88a:57001)

10. C.H. Dowker. On countably paracompact spaces. Canad. J. Math., 3:219-224, 1951. MR 0043446 (13:264c)

11. A. Eden, C. Foias, B. Nicolaenko and R. Temam. Exponential Attractors for Dissipative Evolution Equations. Research in Applied Mathematics Series. John Wiley and Sons, New York, 1994. MR1335230 (96i:34148)

12. C. Foias and E.J. Olson. Finite fractal dimension and Hölder-Lipschitz parametrization. Indiana Univ. Math. J., 45:603-616, 1996. MR1422098 (97m:58120)

13. C. Foias, O. Manley and R. Temam. Modelling of the interaction of small and large eddies in two-dimensional turbulent flows. RAIRO Modél. Math. Anal. Numér., 22(1):93-118, 1988. MR $934703(89 \mathrm{~h}: 76022)$

14. C. Foias, G.R. Sell and R. Temam. Variétés inertielles des équations différentielles dissipatives. C. R. Acad. Sci. Paris I, 301:139-141, 1985. MR801946 (87f:35214a) 
15. C. Foias, G.R. Sell and R. Temam. Inertial manifolds for nonlinear evolutionary equations. J. Differential Equations, 73:309-353, 1988. MR.943945 (89e:58020)

16. B. M. Garay. Strong cellularity and global asymptotic stability. Fund. Math., 138:147-154, 1991. MR:1124542 (92h:54053)

17. R. Geoghegan and R.R. Summerhill. Concerning the shapes of finite-dimensional compacta. Trans. Amer. Math. Soc., 179:281-292, 1973. MR0324637 (48:2987)

18. B. Günther. Construction of differentiable flows with prescribed attractor. Topology Appl., 62:87-91, 1995. MR1318430 (96e:58095)

19. B. Günther and J. Segal. Every attractor of a flow on a manifold has the shape of a finite polyhedron. Proc. Amer. Math. Soc, 119:321-329, 1993. MR.1170545 (93k:54044)

20. P. Hartman. Ordinary Differential Equations. John Wiley and Sons, 1964. MR.0171038 $(30: 1270)$

21. B.R. Hunt and V.Y. Kaloshin. Regularity of embeddings of infinite-dimensional fractal sets into finite-dimensional spaces. Nonlinearity, 12:1263-1275, 1999. MR.1710097(2001a:28009)

22. B.R. Hunt, T. Sauer and J.A. Yorke. Prevalence: A translation-invariant 'almost every' on infinite-dimensional spaces. Bull. Amer. Math. Soc. (N.S.), 27(2):217-238, 1992. MR1161274 (93k:28018)

23. W. Hurewicz and H. Wallman. Dimension Theory. Princeton University Press, Princeton, NJ, 1948. MR0006493 (3:312b)

24. L. Kapitanski and I. Rodnianski. Shape and Morse theory of attractors. Comm. Pure Appl. Math., 53(2):218-242, 2000. MR.1721374 (2000h:37019)

25. R.C. Kirby and L.C. Siebenmann. Foundational Essays on Topological Manifolds, Smoothings and Triangulations. Annals of Mathematical Studies, 88. Princeton University Press, Princeton, NJ, 1977. MR0645390 (58:31082)

26. I. Kukavica. Log-log convexity and backward uniqueness. Proc. Amer. Math. Soc. 135:24152421, 2007. MR.2302562 (2009a:35119)

27. O. Ladyzhenskaya. Attractors for Semigroups and Evolution Equations. Cambridge University Press, Cambridge, 1991. MR1133627 (92k:58040)

28. J. Luukkainen. Assouad dimension: Antifractal metrization, porous sets, and homogeneous measures. J. Korean Math. Soc., 35(1):23-76, 1998. MR.1608518 (99m:54023)

29. R. Mañé. On the dimension of the compact invariant sets of certain nonlinear maps. Springer Lecture Notes in Math., 898:230-242, 1981. MR654892 (84k:58119)

30. S. Mardešić and J. Segal. Shape Theory. North-Holland, 1982. MR676973 (84b:55020)

31. E.J. McShane. Extension of the range of functions. Bull. Amer. Math. Soc. 40:837-842, 1934. MR 1562984

32. A. Norton and C. Pugh. Critical sets in the plane. Michigan Math. J., 38:441-459, 1991. MR.1116500 (92f:57032)

33. E.J. Olson. Bouligand dimension and almost Lipschitz embeddings. Pacific J. Math., 202:459474, 2002. MR1888175 (2003a:37030)

34. E.J. Olson and J.C. Robinson. Almost bi-Lipschitz embeddings and almost homogeneous sets. Trans. Amer. Math. Soc., 362(1):145-168, 2010. MR2550147

35. E. Pinto de Moura and J.C. Robinson. Orthogonal sequences and regularity of embeddings into finite-dimensional spaces. J. Math. Anal. Appl. 368:254-262, 2010a. MR2609274

36. E. Pinto de Moura and J.C. Robinson. Log-Lipschitz continuity of the vector field on the attractor of certain parabolic equations. Submitted, 2010b.

37. J.C. Robinson. Global Attractors: Topology and finite-dimensional dynamics. J. Dynam. Differential Equations, 11(3):557-581, 1999. MR1693866 (2000k:37123)

38. J.C. Robinson. Infinite-dimensional dynamical systems. Cambridge Texts in Applied Mathematics. Cambridge University Press, Cambridge, 2001. MR1881888(2003f:37001a)

39. J.C. Robinson. Linear embeddings of finite-dimensional subsets of Banach spaces into Euclidean spaces. Nonlinearity, 22:711-728, 2009. MR2486352

40. J.C. Robinson. Dimensions, embeddings, and attractors, Cambridge Tracts in Math., 186. Cambridge University Press, Cambridge, 2011.

41. A.V. Romanov. Finite-dimensional limiting dynamics for dissipative parabolic equations. $S b$. Math., 191(3):415-429, 2000. MR.1773256 (2001f:37133)

42. J.M.R. Sanjurjo. On the structure of uniform attractors. J. Math. Anal. Appl., 192:519-528, 1995. MR 1332224 (96c:58109) 
43. E.M. Stein. Singular Integrals and Differentiability Properties of Functions. Princeton University Press, Princeton, NJ, 1982. MR0290095 (44:7280)

44. R. Temam. Infinite-Dimensional Dynamical Systems in Mechanics and Physics, volume 68 of Springer Applied Mathematical Sciences. Springer-Verlag, Berlin, 2nd edition, 1997. MR:1441312(98b:58056)

Mathematical Institute, University of Warwick, Coventry, CV4 7AL, United KingDOM

E-mail address: epmoura@ime.unicamp.br

Mathematical Institute, University of Warwick, Coventry, CV4 7AL, United KingDOM

E-mail address: j.c.robinson@warwick.ac.uk

Mathematical Institute, University of Warwick, Coventry, CV4 7AL, United KingDOM

E-mail address: j.j.sanchez-gabites@warwick.ac.uk 